TI 2008-104/1

Tinbergen Institute Discussion Paper

An Algorithm for Computing the

Nucleolus of Disjunctive Additive

Games with an Acyclic Permission

Structure

René van den Brink ${ }^{7}$

Ilya Katsev²

Gerard van der Laan' 


\section{Tinbergen Institute}

The Tinbergen Institute is the institute for economic research of the Erasmus Universiteit Rotterdam, Universiteit van Amsterdam, and Vrije Universiteit Amsterdam.

Tinbergen Institute Amsterdam

Roetersstraat 31

1018 WB Amsterdam

The Netherlands

Tel.: +31(0)205513500

Fax: $+31(0) 205513555$

Tinbergen Institute Rotterdam

Burg. Oudlaan 50

3062 PA Rotterdam

The Netherlands

Tel.: + $31(0) 104088900$

Fax: $+31(0) 104089031$

Most TI discussion papers can be downloaded at http://www.tinbergen.nl. 


\title{
An algorithm for computing the nucleolus of disjunctive additive games with an acyclic permission structure $^{1}$
}

\author{
René van den Brink ${ }^{2}$ Ilya Katsev ${ }^{3}$ Gerard van der Laan ${ }^{4}$
}

October 31, 2008

\footnotetext{
${ }^{1}$ This research was partly carried out while the second author was visiting the Tinbergen Institute, VU University Amsterdam, on NWO-grant 047.017.017 within the framework of DutchRussian cooperation.

${ }^{2}$ J.R. van den Brink, Department of Econometrics and Tinbergen Institute, VU University, De Boelelaan 1105, 1081 HV Amsterdam, The Netherlands. E-mail: jrbrink@feweb.vu.nl

${ }^{3}$ I.V. Katsev, St. Petersburg Institute for Economics and Mathematics, Russian Academy of Sciences, Tchaikovsky st. 1, 191187 St. Petersburg. E-mail:katsev@yandex.ru

${ }^{4}$ G. van der Laan, Department of Econometrics and Tinbergen Institute, VU University, De Boelelaan 1105, 1081 HV Amsterdam, The Netherlands. E-mail: glaan@feweb.vu.nl
} 


\begin{abstract}
A situation in which a finite set of players can obtain certain payoffs by cooperation can be described by a cooperative game with transferable utility, or simply a TU-game. A (single-valued) solution for TU-games assigns a payoff distribution to every TU-game. A well-known solution is the nucleolus.

A cooperative game with a permission structure describes a situation in which players in a cooperative TU-game are hierarchically ordered in the sense that there are players that need permission from other players before they are allowed to cooperate. The corresponding restricted game takes account of the limited cooperation possibilities by assigning to every coalition the worth of its largest feasible subset.

In this paper we consider the class of non-negative additive games with an acyclic permission structure. This class generalizes the so-called peer group games being nonnegative additive games on a permission tree. We provide a polynomial time algorithm for computing the nucleolus of every restricted game corresponding to some disjunctive nonnegative additive game with an acyclic permission structure. We discuss an application to market situations where sellers can sell objects to buyers through a directed network of intermediaries.
\end{abstract}

Keywords: TU-game, additive game, acyclic permission structure, disjunctive approach, peer group game, nucleolus, algorithm, complexity.

AMS subject classification: $91 \mathrm{~A} 12,5 \mathrm{C} 20$

JEL code: C71 


\section{Introduction}

A cooperative game with transferable utility, or simply a TU-game, is a finite set of players and for any subset (coalition) of players a worth representing the total payoff that the coalition can obtain by cooperating. A payoff vector is a vector which gives a payoff to each of the players, i.e., each component corresponds to precisely one of the players. A payoff vector is efficient if the sum of the payoffs is equal to the worth of the grand coalition consisting of all players. A set-valued solution for TU-games assigns a set of payoff vectors (possibly empty) to every TU-game. A single-valued solution assigns precisely one payoff vector to every TU-game. A solution is said to be efficient if for every game any payoff vector assigned by the solution is efficient. The most well-known efficient set-valued solution is the Core (Gillies, 1953). The two most well-known efficient single-valued solutions are the Shapley value (Shapley, 1953) and the nucleolus (Schmeidler, 1969).

In this paper we assume that the players in a TU-game are part of some hierarchical structure that is represented by a directed graph such that some players need permission from other players before they are allowed to cooperate within a coalition. In the literature two approaches to these games with a permission structure can be found. In the conjunctive approach, as considered in Gilles, Owen and van den Brink (1992) and van den Brink and Gilles (1996), it is assumed that each player needs permission from all its predecessors in the directed graph before it is allowed to cooperate. Alternatively, in the disjunctive approach as developed in Gilles and Owen (1994) and van den Brink (1997), a player needs permission to cooperate of at least one of its direct predecessors (if it has any). So, according to the conjunctive approach a coalition is feasible if and only if for any player in the coalition, all its predecessors are also in the coalition, whereas according to the disjunctive approach a coalition is feasible if and only if for any player in the coalition at least one of its predecessors (if it has any) is also in the coalition. Following an approach similar to that of Myerson (1977) for games with limited communication (graph) structure, in both the conjunctive and disjunctive approach to games with a permission structure a restricted game is derived. In games with a permission structure the restricted game assigns to every coalition the worth of its largest feasible subset. Applying well-known solutions as the Shapley value, Core or nucleolus to such restricted games yields solutions for games with a permission structure.

A special subclass of games with a permission structure arises from peer group situations, as introduced in Brânzei, Fragnelli and Tijs (2002). A peer group situation is a triple consisting of a set of players, a hierarchical structure represented by a rooted (directed) tree, and for each player a non-negative real number representing its potential individual (economic) contribution to the society of all players. This yields an associated TU-game being the additive game in which the worth of any coalition is equal to the sum 
of the individual potentials of its members. In a rooted directed tree there is one top node (not having a predecessor), while any other node has precisely one predecessor. So, in case the hierarchical structure on the player set is a rooted directed tree, the conjunctive approach and the disjunctive approach as described above, coincide. The restricted game of the associated TU-game with respect to such a permission structure is called a peer group game. These peer group games have many interesting applications, see Brânzei et al. (2002). Clearly, in a peer group game the worth of a coalition is the sum of the individual potentials of the members of the largest feasible subset of the coalition. Since the top player is always in this set when it belongs to the coalition, and the largest feasible set is the empty set for any coalition not containing the unique top player, it follows that the top player is a veto player, i.e., any coalition not containing the top player has zero worth in the restricted game. In this paper we generalize peer group games by considering non-negative additive games on acyclic permission structures (which are not necessarily trees). Consequently, these games need not to contain a veto player ${ }^{1}$.

In Arin and Feltkamp (1997) an exponential time algorithm has been given for veto-rich games, i.e., games that have at least one veto player. For the special case of a peer group game a polynomial time algorithm is given in Brânzei, Solymosi and Tijs (2005) and for a class of games satisfying so-called weak digraph monotonicity and weak digraph concavity conditions with respect to acyclic, quasi-strongly connected permission structures, a polynomial time algorithm is given in van den Brink, Katsev and van der Laan (2008). This latter class of games is more general than the class of peer group games, but the acyclic permission structure is still required to be quasi-strongly connected. In this paper we provide a polynomial time algorithm to compute the nucleolus of the restricted game induced by situations in which the associated TU-game is still additive, but in which we allow for any acyclic permission structure, so allowing more than one top player. This considerably widens the applications, for example we can consider situations where sellers can sell objects to buyers through a directed network of intermediaries. Wherease quasistrongly connected networks only can be used for situations with one seller, weakening this by only requiring that the network is acyclic, we can also study such situations with more than one seller. The algorithm presented in this paper computes the nucleolus in polynomial time through a number of iterations. In each iteration a subgame with an acyclic, quasi-strongly connected permission structure is considered and the algorithm developed in van den Brink et al. (2008) is used to compute the nucleolus for this subgame.

This paper is organized as follows. Section 2 is a preliminary section containing cooperative TU-games (with special attention for the nucleolus), directed graphs and games

\footnotetext{
${ }^{1} \mathrm{~A}$ further generalization are the so-called interior operator games where the game is non-negative and additive and the set of feasible coalitions is an antimatroid, see Bilbao et al. (2005, 2008).
} 
with a permission structure. In Section 3 we provide the algorithm to find the nucleolus for disjunctive non-negative additive games with an acyclic permission structure. Section 4 shows that the algorithm indeed computes the nucleolus for this class of games. In Section 5 we discuss some properties of the algorithm, while Section 6 discusses its complexity, showing that it indeed finds the nucleolus in polynomial time. Section 7 illustrates the algorithm with an example of a market situation where sellers can sell objects to buyers through a directed network of intermediaries. Finally, there is an appendix with the algorithm of van den Brink et al. (2008) that is used in each iteration of the algorithm to compute the nucleolus of a non-negative additive subgame with an acyclic, quasi-strongly connected permission structure.

\section{Preliminaries}

\section{$2.1 \quad$ TU-games}

A situation in which a finite set of players can obtain certain payoffs by cooperating can be described by a cooperative game with transferable utility, or simply a TU-game, being a pair $(N, v)$, where $N \subset \mathbb{N}$ is a finite set of $n$ players and $v: 2^{N} \rightarrow \mathbb{R}$ is a characteristic function on $N$ such that $v(\emptyset)=0$. For any coalition $S \subseteq N, v(S)$ is the worth of coalition $S$, i.e., the members of coalition $S$ can obtain a total payoff of $v(S)$ by agreeing to cooperate. For simplicity, for a single player $i$ we denote its worth $v(\{i\})$ by $v(i)$. When there is no confusion about the player set $N$ we often denote the game $(N, v)$ just by its characteristic function $v$. We denote the collection of all characteristic functions on $N$ by $\mathcal{G}^{N}$ and $n=|N|$ denotes the cardinality of $N$. Further, for game $v \in \mathcal{G}^{N}$ and coalition $K \subseteq N$ we define the subgame $v_{K} \in \mathcal{G}^{K}$ by $v_{K}(S)=v(S)$ for all $S \subseteq K$.

A payoff vector for a game is a vector $x \in \mathbb{R}^{n}$ assigning a payoff $x_{i}$ to every $i \in N$. In the sequel, for $S \subseteq N$ we denote $x(S)=\sum_{i \in S} x_{i}$. A payoff vector is efficient if $x(N)=v(N)$ and it is individually rational if $x_{i} \geq v(i)$ for every $i \in N$. The imputation set $I(N, v)$ of game $(N, v)$ is given by

$$
I(N, v)=\left\{x \in \mathbb{R}^{n} \mid x(N)=v(N) \text { and } x_{i} \geq v(i) \text { for every } i \in N\right\},
$$

i.e., $I(N, v)$ is the set of all efficient and individually rational payoff vectors. A (set-valued) solution $F$ on $\mathcal{G}^{N}$ assigns a set $F(N, v) \subset \mathbb{R}^{n}$ of payoff vectors to every characteristic function $v \in \mathcal{G}^{N}$. The most well-known set-valued solution is the Core assigning to every $v \in \mathcal{G}^{N}$ the set

$$
C(N, v)=\{x \in I(N, v) \mid x(S) \geq v(S) \text { for all } S \subset N\}
$$


i.e., it is the set of all imputations that are stable in the sense that no coalition can do better by separating from the grand coalition. The Core of $(N, v)$ is non-empty if and only if the game is balanced, see e.g. Bondareva (1962) or Shapley (1967).

Another (set-valued) solution is the Kernel assigning to every $v \in \mathcal{G}^{N}$ the set $K(N, v)=\left\{x \in I(N, v) \mid\left[s_{i j}(x)=s_{j i}(x)\right]\right.$ or $\left[s_{i j}(x)>s_{j i}(x)\right.$ and $\left.x_{j}=v(j)\right]$ for all $\left.i, j \in N\right\}$, i.e., the set of all imputations such that for each pair of players $i$ and $j$ the complaint $s_{i j}(x)=\max \{v(S)-x(S) \mid i \in S, j \notin S\}$ of $i$ against $j$ is at least equal to the complaint of $j$ against $i$, with equality whenever $j$ gets more than its individual worth $v(j)$.

A solution $F$ is said to be single-valued if it assigns to any $v \in \mathcal{G}^{N}$ a unique payoff vector. One of the two most well-known single-valued solutions is the nucleolus (Schmeidler, 1969). ${ }^{2}$ To define the nucleolus, consider a given characteristic function $v \in \mathcal{G}^{N}$, and let $x \in \mathbb{R}^{n}$ be a payoff vector. Then the excess $e(S, x)$ of a coalition $S \subseteq N$ is defined by

$$
e(S, x)=v(S)-x(S)
$$

Further, let $E(x)$ be the $\left(2^{n}-2\right)$-component vector that is composed of the excesses of all coalitions $S \subset N, S \neq \emptyset$, in a non-increasing order, so $E_{1}(x) \geq E_{2}(x) \geq \ldots \geq$ $E_{2^{n}-2}(x)$. Then the nucleolus $N u c(N, v)$ of the game $(N, v)$ is the unique imputation which lexicographically minimizes the vector-valued function $E(\cdot)$ over the imputation set. Formally,

$$
N u c(N, v)=x \in I(N, v) \text { such that } E(x) \preceq_{L} E(y) \text { for all } y \in I(N, v),
$$

where $\preceq_{L}$ denotes the lexicographic order of vectors. It is well-known that $N u c(N, v) \in$ $K(N, v)$ and that $N u c(N, v) \in C(N, v)$ when $C(N, v) \neq \emptyset$. So, when the game has a nonempty Core, then the nucleolus is in the intersection of the Kernel and the Core.

In a game $(N, v)$, a coalition $S$ is called inessential if it has a partition $\left\{S_{1}, \ldots, S_{r}\right\}$ with $r \geq 2$, such that $v(S) \leq \sum_{j=1}^{r} v\left(S_{j}\right)$. Coalitions which are not inessential are called essential. Notice that single player coalitions are always essential. It is straightforward to observe that for an inessential coalition $S$ it holds that

$$
e(S, x) \leq \sum_{j=1}^{r} e\left(S_{j}, x\right), \text { for all } x \in \mathbb{R}^{n} .
$$

Therefore the Core, and thus also the nucleolus, is independent of inessential coalitions, as was noticed by Huberman (1980). In fact, in any $n$ player game there are at most $(2 n-2)$ coalitions which actually determine the nucleolus, see Brune (1983) and Reijnierse and Potters (1998). However, as noticed by Brânzei et al. (2005), identifying these coalitions is no less laborious as computing the nucleolus itself.

\footnotetext{
${ }^{2}$ The other one is the Shapley value (Shapley, 1953).
} 


\subsection{Directed graphs}

A directed graph or digraph is a pair $(N, D)$ where $N=\{1, \ldots, n\}$ is a finite set of nodes (representing the players) and $D \subseteq N \times N$ is a binary relation on $N$. Given $(N, D)$ and $S \subseteq N$, the graph $(S, D(S))$ is the subgraph on $S$ given by $D(S)=\{(i, j) \in D \mid i, j \in S\}$. In the sequel we simply refer to $D$ for a digraph $(N, D)$ and to $D(S)$ for the subgraph $(S, D(S))$. For $i \in N$ the nodes in $S_{D}(i):=\{j \in N \mid(i, j) \in D\}$ are called the successors of $i$, and the nodes in $P_{D}(i):=\{j \in N \mid(j, i) \in D\}$ are called the predecessors of $i$. For a set $T \subseteq N$, let $S_{D}(T)=\cup_{i \in T} S_{D}(i)$ denote the union of the sets of successors of the players in $T$, respectively $P_{D}(T)=\cup_{i \in T} P_{D}(i)$ the set of all predecessors the players in $T$. Further, $T_{D}=\left\{i \in N \mid P_{D}(i)=\emptyset\right\}$ denotes the set of top nodes in $D$, being the set of nodes not having a predecessor.

For given $D$ on $N$, a path between $i$ and $j$ in $N$ is a sequence of distinct nodes $\left(i_{1}, \ldots, i_{m}\right)$ such that $i_{1}=i, i_{m}=j$, and $\left\{\left(i_{k}, i_{k+1}\right),\left(i_{k+1}, i_{k}\right)\right\} \cap D \neq \emptyset$ for $k=1, \ldots, m-1$. A set of nodes $T \subseteq N$ is connected in digraph $D$ if there is a path between any two nodes in $T$ that only uses arcs between nodes in $T$, i.e., if for every $i, j \in T$ there is a path $\left(i_{1}, \ldots, i_{m}\right)$ between $i$ and $j$ such that $\left\{i_{1}, \ldots, i_{m}\right\} \subseteq T$. A component in $D$ is a maximally connected set $T$ of nodes, i.e., $T$ is connected and $T \cup\{i\}$ is not connected for every $i \in N \backslash T$. A path $\left(i_{1}, \ldots, i_{m}\right)$ from $i_{1}$ to $i_{m}$ is a directed path in $D$ if $\left(i_{k}, i_{k+1}\right) \in D$ for $k=1, \ldots, m-1$. For a set of nodes $T \subset N$ and $i \notin T$, we call a directed path $\left(i_{1}, \ldots, i_{m}\right)$ a path from $T$ to $i$ if $i_{1} \in T$ and $i_{m}=i$. For every (directed) path $p=\left(i_{1}, \ldots i_{m}\right)$ we denote the set of nodes on that path by $H(p)=\left\{i_{1}, \ldots, i_{m}\right\}$. A directed path $\left(i_{1}, \ldots, i_{m}\right), m \geq 1$, in $D$ is a cycle in $D$ if $\left(i_{m}, i_{1}\right) \in D$. We call digraph $D$ acyclic if it does not contain any cycle. Note that acyclicity of a digraph $D$ implies that $D$ is irreflexive, i.e., $(i, i) \notin D$ for all $i \in N$. The collection of all acyclic digraphs on $N$ is denoted by $\mathcal{D}^{N}$. For any $D \in \mathcal{D}^{N}$ the set of top nodes $T_{D}$ is not empty and for every $i \notin T_{D}$ there is a path from $T_{D}$ to $i$. The collection of all paths $p$ from $T_{D}$ to $i \notin T_{D}$ is denoted by $\mathcal{P}_{i}$.

A digraph is called quasi-strongly connected if there exists a node $i_{0} \in N$, such that for every $j \neq i_{0}$ there is a directed path from $i_{0}$ to $j$. When $D$ is, moreover, acyclic then $T_{D}=\left\{i_{0}\right\}$, i.e., $i_{0}$ is the unique top node in $N$. A quasi-strongly connected digraph $D \in \mathcal{D}^{N}$ is a rooted directed tree with the root being the top node $i_{0}$ if there is precisely one path from top-node $i_{0}$ to every other node.

By $\widehat{S}_{D}(i)$ we denote the set of nodes that can be reached from $i$ by a directed path, i.e., $j \in \widehat{S}_{D}(i)$ if and only if there exists a sequence of players $\left(h_{1}, \ldots, h_{t}\right)$ such that $h_{1}=i$, $h_{k+1} \in S_{D}\left(h_{k}\right)$ for all $1 \leq k \leq t-1$, and $h_{t}=j \cdot{ }^{3}$ The players in $\widehat{S}_{D}(i)$ are called the subordinates of $i$, and the players in $\widehat{P}_{D}(i):=\left\{j \in N \mid i \in \widehat{S}_{D}(j)\right\}$ are called the superiors of $i$. For two different nodes $i$ and $j$, we say that node $j \in N$ is a complete subordinate

\footnotetext{
${ }^{3} \mathrm{So}, \widehat{S}_{D}(i)$ is the set of successors of $i$ in the transitive closure of $D$.
} 
of node $i \in N$ in $D$ if every path from $T_{D}$ to $j$ contains node $i$. The set of complete subordinates of node $i$ is denoted by $\bar{S}_{D}(i)$, i.e.,

$$
\bar{S}_{D}(i)=\left\{j \in N \backslash\{i\} \mid i \in H(p) \text { for every } p \in \mathcal{P}_{j}\right\}
$$

Also we call the players in the set

$$
\bar{P}_{D}(i)=\left\{j \in N \backslash\{i\} \mid j \in H(p) \text { for every } p \in \mathcal{P}_{i}\right\}
$$

the complete superiors of $i$ in $D$.

\section{$2.3 \quad$ Restricted games}

In this paper we assume that the players in a TU-game are part of a hierarchical structure that is represented by a directed graph, refered to as a permission structure, such that some players need permission from other players before they are allowed to cooperate within a coalition. A triple $(N, v, D)$ with $(N, v)$ a TU-game and $(N, D)$ a digraph with the player set $N$ as the set of nodes is called a game with a permission structure. In the sequel we assume that $D \in \mathcal{D}^{N}$.

Assumption $2.1(N, D)$ is acyclic.

As noticed in the introduction we can distinguish between the conjunctive and disjunctive approach. In this paper we consider the disjunctive approach as developed in Gilles and Owen (1994) and van den Brink (1997), where a player needs permission to cooperate of at least one of its direct predecessors (if it has any). Therefore a coalition is feasible if and only if for any player in the coalition at least one of its predecessors (if it has any) is also in the coalition. So, for permission structure $(N, D)$, the set of disjunctive feasible coalitions is given by

$$
\Phi^{D}=\left\{S \subseteq N \mid P_{D}(i) \cap S \neq \emptyset \text { for all } i \in S \backslash T_{D}\right\} .
$$

For any $S \subseteq N$, let $\sigma(S)=\cup\left\{T \in \Phi^{D} \mid T \subseteq S\right\}$ be the largest disjunctive feasible subset of $S$ in $D .{ }^{4}$ By Assumption 2.1 we have that for every $S \subseteq N$ with $\sigma(S) \neq \emptyset$, the subgraph $(\sigma(S), D(\sigma(S))$ is acyclic.

Given the triple $(N, v, D)$ with $v \in \mathcal{G}^{N}$ and $D \in \mathcal{D}^{N}$, under the disjunctive permission structure the induced restricted game $r: 2^{N} \rightarrow \mathbb{R}$ is given by

$$
r(S)=v(\sigma(S)) \text { for all } S \subseteq N .
$$

\footnotetext{
${ }^{4}$ Every coalition having a unique largest feasible subset follows from $\Phi^{D}$ being closed under union.
} 
If $\left|T_{D}\right|=1$ then $D$ is quasi-strongly connected and for the unique top player $i_{0}$ it holds that $r(S)=0$ when $i_{0} \notin S$, i.e., the restricted game is a veto-rich game with top-player $i_{0}$ as veto player. ${ }^{5}$ If $D$ is a rooted directed tree, then $\left|P_{D}(i)\right|=1$ for all $i \neq i_{0}$ and the conjunctive and disjunctive approach coincide. In this case the triple $(N, v, D)$ is a peer group situation when the game $(N, v)$ is a non-negative additive game (see Brânzei et al. $(2002)$ ), i.e., there exist non-negative real numbers $a_{i}, i \in N$, such that

$$
v(S)=\sum_{i \in S} a_{i}, S \subseteq N
$$

Then the restricted game $(N, r)$ as defined in (2.1) is a so-called peer group game and is given by

$$
r(S)=v(\sigma(S))=\sum_{\left\{i \in S \mid \widehat{P}_{D}(i) \subseteq S\right\}} a_{i} .
$$

A peer group game $(N, r)$ is a monotone veto-rich game and has a non-empty Core. In particular the payoff vector $x \in \mathbb{R}_{+}^{n}$ given by $x_{i_{0}}=v(N)$ and $x_{i}=0, i \neq i_{0}$, belongs to the Core. On the other hand it holds that $r(S) \leq \sum_{i \in S} a_{i}$ and thus also the payoff vector $x \in \mathbb{R}_{+}^{n}$ given by $x_{i}=a_{i}$ for all $i \in N$ belongs to the Core. As noticed in Brânzei et al. (2002), this class of games with permission structure contains several interesting applications. Also the two games $v$ and its restriction $r$ are games as considered in $\mathrm{Ni}$ and Wang (2007) to study polluted river games. In particular, their LR polluted river is a non-negative additive game, and their DR polluted river game is the restriction $R$ of this game on the permission structure that represents the linear ordering of the players along the river from upstream to downstream.

\section{A polynomial time algorithm for the nucleolus based on quasi-strongly connectedness}

In the remaining of this paper we consider a non-negative additive game with acyclic permission structure $(N, v, D)$. Since $\sigma(S) \subseteq S$ for any $S \subseteq N$, also in this case we have that $r(S)=v(\sigma(S)) \leq \sum_{i \in S} a_{i}$. Therefore the payoff vector $x \in \mathbb{R}_{+}^{n}$ given by $x_{i}=a_{i}$ for all $i \in N$ is in the Core and thus the Core is nonempty and contains the nucleolus.

We first show that for any non-negative additive game with acyclic permission structure $(N, v, D)$, there exists a subset $K \subseteq N$ with the properties that (i) the subgraph $(K, D(K))$ is an acyclic, quasi-strongly connected permission structure and (ii)

\footnotetext{
${ }^{5} \mathrm{~A}$ game $(N, v)$ is a veto-rich game if it contains a veto player being a player $i$ such that $v(S)=0$ when $i \notin S$.
} 
$\sum_{j \in K} x_{j}=\sum_{j \in K} a_{j}$ for any payoff vector $x \in \mathbb{R}^{n}$ in the Core of the restricted game $(N, r)$, so also for the nucleolus. These properties play an important role in an algorithm to compute the nucleolus of the restricted game obtained from a non-negative additive game with acyclic permission structure $(N, v, D)$. In fact the nucleolus is obtained by computing the nucleolus of a sequence of smaller non-negative additive games with quasistrongly connected permission structures. By definition the properties hold for $K=N$ when the graph has only one top player. So, in the sequel of this section we only consider the case that $\left|T_{D}\right| \geq 2$. The first lemma is obvious and stated without proof.

Lemma 3.1 For every acyclic permission structure $(N, D)$ it holds: $j$ is a (complete) superior of $i$ if and only if $i$ is a complete subordinate of $j$.

For a top player $t \in T_{D}$, let the set $U^{t}$ be defined by $\bar{S}_{D}(t) \cup\{t\}$, i.e., $U^{t}$ contains top player $t$ together with all its complete subordinates. Observe that $T_{D} \backslash U^{t} \neq \emptyset$, because $\left|T_{D}\right| \geq 2$ and $\left(T_{D} \backslash\{t\}\right) \cap U^{t}=\emptyset$. Further, for $i \in \bar{S}_{D}(t)$, define

$$
U_{i}=\cup\{H(p) \mid p \text { is a directed path from } t \text { to } i\},
$$

i.e., the set $U_{i} \subset N$ is the union of all players on all directed paths from top player $t$ to its complete subordinate $i$. Then we have the next lemma.

Lemma 3.2 Let $(N, D)$ be an acyclic permission structure, $t \in T_{D}$ a top player, and $i \in \bar{S}_{D}(t)$ a complete subordinate of $t$. Then $U_{i} \subseteq U^{t}$.

Proof. By definition we have that $t \in U^{t}$. Since $t$ is a complete superior of $i$, it follows that $t$ is a complete superior of any $h \in U_{i} \backslash\{t\}$. Suppose not. Then for some $h \in U_{i} \backslash\{t\}$, there is a path from a top player $t^{\prime} \neq t$ to $h$, and so also a path from $t^{\prime}$ to $i$, contradicting that $t$ is a complete superior of $i$. By Lemma 3.1 we have that any $h$ in $U_{i}$ is a complete subordinate of $t$, and thus $h \in U^{t}$.

Since $U_{i} \subseteq U^{t}$ for all $i \in U^{t}=\bar{S}_{D}(t) \cup\{t\}$ (see Lemma 3.2) it follows that

$$
U^{t}= \begin{cases}\{t\} & \text { if } \bar{S}_{D}(t)=\emptyset, \\ \cup_{\left\{i \in \bar{S}_{D}(t)\right\}} U_{i} & \text { otherwise. }\end{cases}
$$

So, $U^{t}$ is the union of all sets $U_{i}$ of the complete subordinates of top player $t$ when $t$ has at least one complete subordinate, and $U^{t}=\{t\}$ otherwise.

The next proposition shows the existence of a subset $K \subseteq N$ such that the subgraph $(K, D(K))$ is an acyclic, quasi-strongly connected permission structure.

Proposition 3.3 Let $(N, D)$ be an acyclic permission structure. Then for every $t \in T_{D}$ it holds that the subgraph $\left(U^{t}, D\left(U^{t}\right)\right)$ is an acyclic, quasi-strongly connected permission structure with $t$ its unique top node. 
Proof. First, when $\bar{S}_{D}(t)=\emptyset$, then $U^{t}=\{t\}$ and the statement is true. Otherwise, let $i$ be a complete subordinate of $t$. Obviously $t$ is the unique top-player in the subgraph $\left(U_{i}, D\left(U_{i}\right)\right)$. Further, the subgraph $\left(U_{i}, D\left(U_{i}\right)\right)$ is acyclic and quasi-strongly connected. Acyclicity follows from the acyclicity of $D$ and the fact that $D\left(U_{i}\right) \subseteq D$. Quasi-strongly connectedness follows from acyclicity of $D$ and the fact that $U_{i}$ is the union of all directed paths from $t$ to $i$. Then the result follows because $U^{t}=\cup_{\left\{i \in \bar{S}_{D}(t)\right\}} U_{i}$.

The next lemma states that when $N$ is partitioned in two disjunctive feasible sets, for both sets it holds that the total payoff of its players in every core payoff vector of the restricted game is equal to their own value.

Lemma 3.4 Let $A, B \in \Phi^{D}$ be two disjunctive feasible coalitions in a non-negative additive game with acyclic permission structure $(N, v, D)$ such that $A \cap B=\emptyset$ and $A \cup B=N$. Then $x(A)=\sum_{i \in A} a_{i}$ and $x(B)=\sum_{i \in B} a_{i}$ for every core element $x \in C(N, r)$.

Proof. By definition of the restricted game $(N, r)$ and feasibility of $A$ we have that $r(A)=v(\sigma(A))=v(A)=\sum_{i \in A} a_{i}$ and $r(B)=v(\sigma(B))=v(B)=\sum_{i \in B} a_{i}$. Now, let $x \in C(N, r)$. Then $x(A) \geq r(A)$ and $x(B) \geq r(B)$. Form the second inequality we obtain that that $x(A)=r(N)-x(B) \leq \sum_{i \in N} a_{i}-\sum_{i \in B} a_{i}=\sum_{i \in A} a_{i}=r(A)$. Hence $x(A)=r(A)=\sum_{i \in A} a_{i}$. Analogous $x(B)=r(B)=\sum_{i \in B} a_{i}$.

We now state the final result of this section.

Proposition 3.5 Let $(N, D)$ be an acyclic permission structure and $t \in T_{D}$. Then $x\left(U^{t}\right)=$ $\sum_{i \in U^{t}} a_{i}$ and $x\left(N \backslash U^{t}\right)=\sum_{i \in N \backslash U^{t}} a_{i}$ for every core element $x \in C(N, r)$.

Proof. By definition we have that $U^{t}$ is disjunctive feasible. To show that also $N \backslash U^{t}$ is disjunctive feasible, consider a player $i \in N \backslash U^{t}$. Since $i \neq t$ and $i$ is also not a complete subordinate of $t$, there is a path from $T_{D} \backslash\{t\}$ to $i$. Hence $N \backslash U^{t}$ is disjunctive feasible. So, both $U^{t}$ and $N \backslash U^{t}$ are disjunctive feasible and thus the proposition follows by applying Lemma 3.4 to $A=U^{t}$ and $B=N \backslash U^{t}$.

From the Propositions 3.3 and 3.5 it follows that for any $t \in T_{D}$ it holds that $U^{t} \subset N$ satisfies both properties that $\left(U^{t}, D\left(U^{t}\right)\right)$ is an acyclic, quasi-strongly connected permission structure and $\sum_{j \in U^{t}} x_{j}=r\left(U^{t}\right)=\sum_{j \in U^{t}} a_{j}$ for every $x \in C(N, r)$, so also for the nucleolus. This property is now used to compute the nucleolus of the restricted game $(N, r)$ obtained from a non-negative additive game with acyclic permission structure (that is not necessarily quasi-strongly connected) in a finite number of steps. At each step we compute the nucleolus of a smaller additive game with an acyclic, quasi-strongly connected permission structure by applying the $\mathcal{O}\left(n^{4}\right)$ algorithm of van den Brink et al. (2008) for 
such games. This algorithm is an adaptation of the algorithm of Arin and Feltkamp (1997), which computes the nucleolus of veto-rich games in exponential time. Note that permission games with an acyclic, quasi-strongly connected permission structure are indeed veto-rich games, which is not true for the ive the algorithm, we introduce one more notation. Let $(N, D)$ be an acyclic permission structure, $t \in T_{D}$ be one of the top players and $K=N \backslash U^{t}$. Then the set $D^{K} \in \mathcal{D}^{K}$ on the set of players $K$ is given by

$$
(i, j) \in D^{K} \text { if and only if }(i, j) \in D \text { and } P_{D}(j) \cap U^{t}=\emptyset \text {. }
$$

So, for two players $i, j \in K,(i, j)$ is an arc in $D^{K}$ if and only if $(i, j)$ is an arc in $D$ and $j$ does not have a predecessor in $U^{t}$. Stated differently, $D^{K}$ contains all $\operatorname{arcs}$ in $D(K)$, except the $\operatorname{arcs}(i, j)$ such that $j$ is a successor of top player $t$ or of one of its complete subordinates. Finally, we assume that the players are enumerated (labeled) by the numbers $1,2, \ldots, n$ in such a way that for any $i, j \in\{1, \ldots, n\}$ it holds that $i<j$ if $(i, j) \in D$. From the theory on acyclic directed graphs it is well-known that such a enumeration exists. Observe that this assumption implies that node $1 \in T_{D}$.

\section{Algorithm}

Step 1 Set $k=1, N_{1}=N, D_{1}=D$ and $t_{1}=1$. Goto Step 2 .

Step 2 Consider the non-negative additive game with acyclic, quasi-strongly connected permission structure $\left(U^{t_{k}}, v_{k}, D_{k}\left(U^{t_{k}}\right)\right)$ with

$$
v_{k}(U)=v(U) \text { for all } U \subseteq U^{t_{k}} .
$$

Let $r_{k}$ be the restricted game of $\left(U^{t_{k}}, v_{k}, D_{k}\left(U^{t_{k}}\right)\right)$. Goto Step 3.

Step 3 Apply the (polynomial time) algorithm of van den Brink et al. (2008) (see the appendix) to find the nucleolus of the restricted game $\left(U^{t_{k}}, r_{k}\right)$. Assign $y_{i}=$ $N u c_{i}\left(U^{t_{k}}, r_{k}\right)$ to every $i \in U^{t_{k}}$. Goto Step 4 .

Step 4 If $U^{t_{k}}=N_{k}$ then Stop. Otherwise, goto Step 5 .

Step 5 Define $N_{k+1}=N_{k} \backslash U^{t_{k}}$ and $D_{k+1} \in \mathcal{D}^{N_{k+1}}$ by $D_{k+1}=D_{k}^{N_{k+1}}$ (i.e., $D_{k+1}=D^{K}$ as defined in formula (3.2) with $D=D_{k}$ and $K=N_{k} \backslash U^{t_{k}}$ ). Define $t_{k+1} \in T_{D_{k+1}}$ as the top player in $D_{k+1}$ with the lowest label $\left(t_{k+1} \leq h\right.$ for every $\left.h \in T_{D_{k+1}}\right)$. Consider the set $U^{t_{k+1}}$ consisting of $t_{k+1}$ and all its complete subordinates in the graph $\left(N_{k+1}, D_{k+1}\right)$. Set $k=k+1$ and return to step 2 . 


\section{The algorithm works}

In this section we prove that the algorithm indeed finds the nucleolus of the original nonnegative additive game with acyclic permission structure $(N, v, D)$. As a first observation, according to Proposition 3.5 it must hold that $\sum_{i \in U^{1}} N u c_{i}(N, r)=\sum_{i \in U^{1}} a_{i}$, so the total payoff $\sum_{i \in U^{1}} \quad a_{i}$ assigned in the first iteration of the algorithm to the players in $U^{1}$ is indeed equal to the total payoff that the players in $U^{1}$ attain at the $N u c(N, r)$. Of course, we still have to prove that the individual payoffs assigned at the first iteration are the individual payoffs for the players in $U^{1}$ in $N u c(N, r)$, and subsequently for the payoffs assigned at any next iteration. This will be proved by using the Davis-Maschler reduced game property. For a game $(N, v)$, let $T \subset N$ be a nonempty coalition and $x \in \mathbb{R}^{n}$ a payoff vector. Then the Davis-Maschler reduced game on $T$ at $x$ is the game $\left(T, v_{T}^{x}\right)$ given by $v_{T}^{x}(T)=v(N)-x(N \backslash T)$ and $v_{T}^{x}(S)=\max _{Q \subseteq N \backslash T}(v(S \cup Q)-x(Q)), S \subset T$, $S \neq T$. Observe that in the definition of the reduced game only the values $x_{j}$ of the players $j \in N \backslash T$ appear.

\section{Property 4.1 Davis-Maschler reduced game property}

For a game $(N, v)$, let $x$ be the Nucleolus of $(N, v)$. Then for any $T \subset N$ it holds that

$$
N u c_{i}(N, v)=N u c_{i}\left(T, v_{T}^{x}\right), \text { for all } i \in T \text {. }
$$

In the sequel we will denote the characteristic function of the Davis-Maschler reduced game with respect to the nucleolus $x=N u c(N, v)$ and coalition $T \subset N$ just by $v^{\prime}$, if there is no confusion ${ }^{6}$. Recall that for game $v \in \mathcal{G}^{N}$ and coalition $T \subset N$, the subgame $v_{T} \in \mathcal{G}^{T}$ is given by $v_{T}(S)=v(S)$ for all $S \subseteq T$. We now have the following proposition with respect to $U^{t}$ for some $t \in T_{D}$. For notational simplicity, in the following we denote $U^{t}=K$.

Proposition 4.2 For a non-negative additive game with acyclic permission structure $(N, v, D)$ with $\left|T_{D}\right| \geq 2$, let $t \in T_{D}$ be a top player and $K=U^{t}$. Then the Davis-Maschler reduced game $\left(K, r^{\prime}\right)$ of the restricted game $(N, r)$ with respect to the nucleolus $x \in \mathbb{R}^{n}$ and the set $K$, coincides with the subgame $\left(K, r_{K}\right)$ of $(N, r)$.

Proof. First, observe that $K$ is feasible and thus $r_{K}(K)=\sum_{i \in K} a_{i}$. On the other hand $r^{\prime}(K)=r(N)-x(N \backslash K)=\sum_{i \in N} a_{i}-\sum_{i \in N \backslash K} a_{i}=\sum_{i \in K} a_{i}$, where the first equality is by definition of the reduced game and the second equality by Proposition 3.5. So, $r_{K}(K)=r^{\prime}(K)=\sum_{i \in K} a_{i}$.

\footnotetext{
${ }^{6}$ In general, the Davis-Maschler reduced game property is stated for an arbitrary solution. Since we apply it here to compute the nucleolus, we only state it in terms of this particular solution.
} 
Next, consider set $U \subset K$. By definition of the Davis-Maschler reduced game we have

$$
r^{\prime}(U)=\max _{S \subseteq N \backslash K}(r(U \cup S)-x(S)) .
$$

We first show that $r^{\prime}(U)=r^{\prime}(\sigma(U))$. Therefore it is sufficient to show that for any $S \subseteq$ $N \backslash K$ it holds that $\sigma(U \cup S)=\sigma(\sigma(U) \cup S)$, because for any $S \subseteq N \backslash K$ the value of $r(U \cup S)$ in equation (4.4) is equal to the worth of $\sigma(U \cup S)$ in game $v$. Since $\sigma(U) \subseteq U$, it is evident that $\sigma(\sigma(U) \cup S) \subseteq \sigma(U \cup S)$. Suppose that the inclusion does not hold the other way around, i.e., there exists some player $i \in \sigma(U \cup S) \backslash \sigma(\sigma(U) \cup S)$. For this player there is a path $p$ from $T_{D}$ to $i$ consisting of players in $\sigma(U \cup S)$ only, and there does not exist a path from $T_{D}$ to $i$ consisting of players in $\sigma(\sigma(U) \cup S)$ only. Consider a path $p=\left(p_{1}, \ldots, p_{m}\right)$ from $T_{D}$ to $i$ with $H(p) \subseteq \sigma(U \cup S)$. We distinguish the following two cases:

(i). Suppose that $p$ is a directed path from $t$ to $i$. Since $P_{D}(j) \subset K$ for all $j \in K$, there is a $k \in\{2, \ldots, m\}$ such that $\left\{p_{1}, \ldots p_{k}\right\} \subseteq U$ and $\left\{p_{k+1}, \ldots p_{m}\right\} \subseteq S$. Since $\left(p_{1}, \ldots p_{k}\right)$ is a directed path in $U$, we have that $\left\{p_{1}, \ldots p_{k}\right\} \subseteq \sigma(U)$. Thus, $H(p) \subseteq \sigma(U) \cup S$. But then $H(p) \subseteq \sigma(\sigma(U) \cup S)$ since $p$ is a directed path with $p_{1}=t$.

(ii). Suppose that $p$ is a directed path from another top-player $t^{\prime} \neq t$ to $i$. Since players in $K=U^{t}$ do not have predecessors in $N \backslash K$, we have that $U \cap H(p)=\emptyset$. Thus, $H(p) \subseteq \sigma(S)$, and so $H(p) \subseteq \sigma(\sigma(U) \cup S)$.

From (i) and (ii) we conclude that $\sigma(U \cup S)=\sigma(\sigma(U) \cup S)$. Hence we have that $r^{\prime}(U)=$ $r^{\prime}(\sigma(U))$ and it remains to prove that $r_{K}(U)=r^{\prime}(U)$ for any feasible $U \subset K$.

Observe that $r_{K}(U)=\sum_{i \in U} a_{i}$ when $U$ is feasible. To find $r^{\prime}(U)$, we first show that for finding the maximum in (4.4), it is sufficient to consider only sets $S$ such that $U \cup S$ is feasible. If $U \cup S$ is not feasible, then

$$
\begin{aligned}
& r(U \cup S)-x(S)=r(\sigma(U \cup S))-x(S) \leq \\
& r(U \cup(\sigma(U \cup S) \backslash U))-x(\sigma(U \cup S) \backslash U)-x(S \backslash(\sigma(U \cup S) \backslash U)) \leq \\
& r(U \cup(\sigma(U \cup S) \backslash U))-x(\sigma(U \cup S) \backslash U),
\end{aligned}
$$

where the first inequality follows from $\sigma(U \cup S) \backslash U \subseteq S$ and $\sigma(U \cup S) \backslash U \neq S$ if $U \cup S$ is not feasible. So, in case that $U \cup S$ is not feasible, replacing set $S$ by set $\sigma(U \cup S) \backslash U$ does not decrease $r(U \cup S)-x(S)$ in formula (4.4), and thus this expresion is maximized by a coalition $S$ such that $U \cup S$ is feasible.

By definition of $r^{\prime}$, it now follows that there is some $S \subseteq N \backslash K$ such that $U \cup S$ is feasible and

$$
r^{\prime}(U)=r(U \cup S)-x(S)=\sum_{i \in U \cup S} a_{i}-x(S) .
$$


Further, since $U \cup S$ is feasible and $U \subset K=U^{t}$, we have that also $K \cup S$ is feasible. So, by the fact that $x \in C(N, r)$, we have that $x(K \cup S)=x(K)+x(S) \geq \sum_{i \in K \cup S} a_{i}$. By Proposition 3.5 we have that $x(K)=a(K)$ and thus $x(S) \geq \sum_{i \in S} a_{i}$. It follows with equation (4.5) that

$$
r^{\prime}(U)=\sum_{i \in U} a_{i}+\sum_{i \in S} a_{i}-x(S) \leq \sum_{i \in U} a_{i}=r(U) .
$$

From (4.6) and the fact that by definition of the reduced game $r^{\prime}(U) \geq r(U)$, we conclude that

$$
r^{\prime}(U)=r(U)=\sum_{i \in U} a_{i}
$$

This proves that $r^{\prime}(U)=r_{K}(U)$ for all $U \subset K=U^{t}$.

In the first iteration the algorithm finds the nucleolus of $\left(U^{1}, r_{1}\right)$. Clearly, the restricted game $\left(U^{1}, r_{1}\right)$ of $\left(U^{1}, v_{1}, D_{1}\right)$ is equal to the subgame $\left(U^{1}, r_{U^{1}}\right)$ of $(N, r)$, which is equal to the Davis-Maschler reduced game according to the proposition above. So, with the Davis-Maschler reduced game Property 4.1, the proposition above shows that in the first iteration the algorithm indeed computes the nucleolus payoffs of the players in $U^{1}$ in game $(N, r)$. For $t \in T_{D}$ and $K=N \backslash U^{t}$, the next proposition shows that the DavisMaschler reduced game with respect to the nucleolus and the set $K$ coincides with the game $\left(K, r_{2}\right)$, where $r_{2}$ is the restricted game of the non-negative game with the reduced acyclic permission structure $\left(K, v_{K}, D^{K}\right)$ on the set of players $K$, where $\left(K, v_{K}\right)$ is the subgame of $(N, v)$ on $K$ and $D^{K}$ is as given in formula (3.2) for $t=1$, i.e., $r_{2}$ is the restricted game used in the second iteration of the algorithm.

Proposition 4.3 For a non-negative additive game with acyclic permission structure $(N, v, D)$ with $\left|T_{D}\right| \geq 2$, let $t \in T_{D}$ and $K=N \backslash U^{t}$. Then the Davis-Maschler reduced game $\left(K, r^{\prime}\right)$ of the restricted game $(N, r)$ with respect to the nucleolus payoff vector $x \in \mathbb{R}^{n}$ and the set $K$, coincides with the restricted game $\left(K, r_{2}\right)$ of the non-negative additive game with acyclic permission structure $\left(K, v_{K}, D^{K}\right)$ with $D^{K}$ as defined in formula (3.2).

Proof. By definition, $P_{D^{K}}(j) \subseteq K$ for all $j \in K$, so $K$ is feasible in the reduced graph $\left(K, D^{K}\right)$. Hence $r_{2}(K)=\sum_{i \in K} a_{i}$. On the other hand, $r^{\prime}(K)=r(N)-x(N \backslash K)=$ $r(N)-x\left(U^{t}\right)=\sum_{i \in N} a_{i}-\sum_{i \in U^{t}} a_{i}=\sum_{i \in K} a_{i}$, where the first equality is by definition of the reduced game and the second equality by Proposition 3.5. So, $r_{2}(K)=r^{\prime}(K)$.

Next, for a set $S \subset K$ we consider two cases.

(i). First, suppose that $P_{D}(S) \cap U^{t}=\emptyset$. For all $U \subseteq U^{t}$ it holds that $\sigma(U \cup S)=\sigma(U) \cup \sigma(S)$, since $P_{D}(U) \cap S=\emptyset$. So,

$$
r(S \cup U)-x(U)=v(\sigma(U) \cup \sigma(S))-x(U)=
$$




$$
v(\sigma(U))+v(\sigma(S))-x(U)=r(S)+r(U)-x(U) \leq r(S),
$$

where the second equality follows from $v$ being an additive game and the (last) inequality follows from the nucleolus being a core element. Hence

$$
r^{\prime}(S)=\max _{U \subseteq U^{t}}(r(S \cup U)-x(U))=r(S) .
$$

On the other hand, for all $i, j \in S$ we have that $(i, j) \in D^{K}$ if $(i, j) \in D$, because no player $j \in S$ has a predecessor in $U^{t}$. Hence, $\sigma(S)=\sigma_{K}(S)$, where $\sigma_{K}(S)$ is the largest feasible subset of $S$ in $\left(K, D_{K}\right)$. So,

$$
r^{\prime}(S)=r(S)=v(\sigma(S))=v\left(\sigma_{K}(S)\right)=r_{2}(S) .
$$

(ii). Secondly we consider the case that $P_{D}(S) \cap U^{t} \neq \emptyset$. Now, let $S^{\prime} \subseteq S$ be given by $S^{\prime}=\sigma\left(U^{t} \cup S\right) \backslash U^{t}$. By definition of $\sigma, U^{t} \cup S^{\prime}$ is the maximal feasible subset of $U^{t} \cup S$ and thus

$$
r\left(U^{t} \cup S\right)-x\left(U^{t}\right)=v\left(U^{t} \cup S^{\prime}\right)-x\left(U^{t}\right)=\sum_{i \in U^{t} \cup S^{\prime}} a_{i}-\sum_{i \in U^{t}} a_{i}=\sum_{i \in S^{\prime}} a_{i},
$$

where the second equality follows by Proposition 3.5. Hence we have that

$$
r^{\prime}(S)=\max _{U \subseteq U^{t}}(r(S \cup U)-x(U)) \geq r\left(U^{t} \cup S\right)-x\left(U^{t}\right)=\sum_{i \in S^{\prime}} a_{i} .
$$

We now show that this holds with equality. First, recall from the proof of Proposition 4.2 that $\sigma(U \cup S)=\sigma(\sigma(U) \cup S)$ for every $U \subset U^{t}$ and $S \subset K$. Hence

$$
r(U \cup S)-x(U)=r(\sigma(U) \cup S)-x(U) \leq r(\sigma(U) \cup S)-x(\sigma(U)),
$$

so that $\max _{U \subseteq U^{t}}(r(S \cup U)-x(U))$ will be obtained by a feasible set $U$. Suppose there exists a feasible $U \subseteq U^{t}$ with $r(U \cup S)-x(U)>\sum_{i \in S^{\prime}} a_{i}$. Then, with $S_{U} \subseteq S$ given by $S_{U}=\sigma(U \cup S) \backslash U$, it follows in an analogous way as for $U=U^{t}$ above, that $\sigma(U \cup S)=$ $U \cup S_{U}$, and thus

$$
\begin{aligned}
& r(U \cup S)-x(U)=v\left(U \cup S_{U}\right)-x(U)=\sum_{i \in U \cup S_{U}} a_{i}-x(U)= \\
& \sum_{i \in S_{U}} a_{i}+\sum_{i \in U} a_{i}-x(U) \leq \sum_{i \in S_{U}} a_{i} \leq \sum_{i \in S^{\prime}} a_{i},
\end{aligned}
$$

where the first inequality follows because $U$ is feasible and the nucleolus lies in the core, and the second inequality because $U \subseteq U^{t}$ and thus $S_{U}=\sigma(U \cup S) \backslash U \subseteq \sigma\left(U^{t} \cup S\right) \backslash U^{t}$. From equations (4.7) and (4.8) it follows that

$$
r^{\prime}(S)=\sum_{i \in S^{\prime}} a_{i}=v\left(S^{\prime}\right)
$$


It remains to prove that also $r_{2}(S)=v\left(S^{\prime}\right)$. By definition we have that $r_{2}(S)=$ $v_{K}\left(\sigma_{K}(S)\right)=v\left(\sigma_{K}(S)\right)$, where $\sigma_{K}(S)$ is the maximal feasible subset of $S$ in graph $\left(K, D^{K}\right)$. So, it remains to show that $\sigma_{K}(S)=S^{\prime}$.

We first show that $\sigma_{K}(S) \subseteq S^{\prime}=\sigma\left(U^{t} \cup S\right) \backslash U^{t}$. Consider $i \in \sigma_{K}(S)$. For such a player $i$ there is a path $p$ from $T_{D^{K}}$ to $i$ which only contains elements of $S$. We consider two cases. If $p$ is a path from a top $t^{\prime} \in T_{D}$, then $i \in \sigma(S) \subseteq S^{\prime}$. For the case that $p$ is a path from a top $t^{\prime}$ in $T_{D^{K}} \backslash T_{D}$, then $t^{\prime}$ is a top in $\left(K, D^{K}\right)$ but not in $(N, D)$ and thus $P^{D}\left(t^{\prime}\right) \cap U^{t} \neq \emptyset$, implying that in $(N, D)$ there is a path $p^{\prime}$ from $t$ to $t^{\prime}$. Hence, the path $p^{\prime \prime}$ consisting of the path $p^{\prime}$ from $t$ to $t^{\prime}$ and the path $p$ from $t^{\prime}$ to $i$ is a path in $(N, D)$ from $T_{D}$ to $i$. Since $H\left(p^{\prime}\right) \backslash\left\{t^{\prime}\right\} \subseteq U^{t}$ and $H(p) \subseteq S$, we have that the set of nodes $H\left(p^{\prime \prime}\right) \subseteq U^{t} \cup S$ and thus $H\left(p^{\prime \prime}\right) \subseteq \sigma\left(U^{t} \cup S\right)$. So $i \in \sigma\left(U^{t} \cup S\right)$ and we can conclude that $i \in \sigma\left(U^{t} \cup S\right) \backslash U^{t}=S^{\prime}$.

Next we show the reverse that $S^{\prime} \subseteq \sigma_{K}(S)$. Let $i \in S^{\prime}$. Then $i \in \sigma\left(U^{t} \cup S\right)$ and thus there is path $p$ from $T_{D}$ to $i$ that consists of elements of $U^{t} \cup S$. Again there are two cases. If $H(p) \subseteq S$, then $i \in \sigma(S) \subseteq \sigma_{K}(S)$. Otherwise, $p$ consists of two subpaths $p^{\prime}$ in $U^{t}$ and $p^{\prime \prime}$ in $S$ that are connected to each other by a link from the last node of $p^{\prime}$ to the first node of $p^{\prime \prime}$. Let $j$ be that last node of path $p^{\prime \prime}$ that has a predecessor in $U^{t}$. Then, by construction, $j$ is a top-node in game $\left(K, D^{K}\right)$ and thus the part of $p^{\prime \prime}$ from $j$ to $i$ is a path in $\left(K, D_{K}\right)$ from $T_{D^{K}}$ to $i$. So $i \in \sigma_{K}(S)$.

By repeated application of the propositions above it follows that the algorithm of Section 3 computes the nucleolus of $(N, r)$.

Proposition 4.4 For a non-negative additive game with acyclic permission structure $(N, v, D)$, the algorithm described in Section 4 finds the nucleolus of $(N, r)$ within a finite number of iterations.

Proof. In the first iteration the algorithm finds the nucleolus of the restricted game $\left(U^{1}, r_{1}\right)$ of $\left(U^{1}, v_{1}, D_{1}\right)$, which is equal to the subgame $\left(U^{1}, r_{U^{1}}\right)$ of $(N, r)$. By Proposition 4.2 and the Davis-Maschler reduced game property it follows that $N u c_{i}\left(U^{1}, r_{1}\right)=N u c_{i}(N, r)$ for all $i \in U^{1}$. If $U^{1}=N$, the algorithm ends with the nucleolus in one iteration. Otherwise, the algorithm continues in iteration 2 with the restricted game $\left(N \backslash U^{1}, r_{2}\right)$ of $\left(N \backslash U^{1}, v, D_{2}\right)$. By Proposition 4.3 this restricted game is equal to the DM reduced game on $N \backslash U^{1}$ with respect to the nucleolus and so the nucleolus payoffs of the game $\left(N \backslash U^{1}, r_{2}\right)$ are equal to the nucleolus payoffs of the players in $N \backslash U^{1}$ in the game $(N, r)$. Repeating the arguments of the first iteration, in the second iteration the algorithm computes the nucleolus payoffs of the game $\left(N \backslash U^{1}, r_{2}\right)$ for the players $i \in U^{t_{2}}$, which thus is equal to their nucleolus payoffs in the game $(N, r)$. Subsequentially in each iteration $k$ the algorithm computes the nucleolus payoffs of the set of players in $U^{t_{k}}$. Since the number of players is reduced 
with at least one in each iteration of the algorithm, the algorithm ends within at most $n$ iterations.

\section{Properties of the algorithm}

In this section we first show several properties of the algorithm. From these properties we then obtain an interesting property of the nucleolus of a non-negative additive game with permission structure, namely that each coalition consisting of a free player and its complete subordinates distributes its own value among themselves. A free player is a player that does not have a complete superior.

Definition 5.1 A player $i \in N$ in an acyclic permission structure $(N, D)$ is a free player if and only if $\bar{P}_{D}(i)=\emptyset$.

Example 5.2 Consider permission structure $(N, D)$ with $N=\{1,2,3,4,5,6\}$ and $D=$ $\{(1,3),(1,4),(2,4),(3,5),(3,6),(4,6)\}$. This permission structure has four free players. Two of them are the two top players 1 and 2. Besides the top players the two other free players are 4 and 6 .

In the sequel we denote the set of free players in $(N, D)$ by $F_{D}$. Notice that $T_{D} \subseteq F_{D}$. Recall from Subsection 2.2 that for every non-top player $i$, the set $\mathcal{P}_{i}$ denotes the collection of all paths from $T_{D}$ to $i$. For a path $p=\left(i_{1}, \ldots, i_{m-1}, i_{m}\right) \in \mathcal{P}_{i}\left(\right.$ so $i_{1} \in T_{D}$ and $i_{m}=i$ ), in the sequel $H_{i}(p)$ denotes the set of players $H(p) \backslash\{i\}$, i.e., $H_{i}(p)$ is the set of all players on the path $p$ except player $i$ itself. We now give two lemmas. The first one is obvious and stated without proof.

Lemma 5.3 For every acyclic permission structure $(N, D)$, if $j$ is a (complete) superior of $i$ and $k$ is a (complete) superior of $j$ then $k$ is a (complete) superior of $i$.

The second lemma states that for any free player $i \in F_{D} \backslash T_{D}$ there exist (at least) two paths $p$ and $q$ in $\mathcal{P}_{i}$ such that $H_{i}(p) \cap H_{i}(q)=\emptyset$, i.e., for any non-top free player $i$ there exist two disjunct paths (except for $i$ itself) from $T_{D}$ to $i$. Recall that it is assumed that the players are enumerated (labeled) by the numbers $1,2, \ldots, n$ in such a way that for any $i, j \in\{1, \ldots, n\}$ it holds that $i<j$ if $(i, j) \in D$.

Lemma 5.4 Let $(N, D)$ be an acyclic permission structure and let $i$ be a player in $F_{D} \backslash T_{D}$. Then there exist (at least) two paths $p$ and $q$ in $\mathcal{P}_{i}$, such that $H_{i}(p) \cap H_{i}(q)=\emptyset$. 
Proof. For any two different paths $p^{h}$ and $p^{k}$ in $\mathcal{P}_{i}$, define $m_{h k}=\max \left\{j \mid j \in H_{i}\left(p^{k}\right) \cap\right.$ $\left.H_{i}\left(p^{h}\right)\right\}$ with the convention that $m_{h k}=0$ if $H_{i}\left(p^{k}\right) \cap H_{i}\left(p^{h}\right)=\emptyset$, i.e., $m_{h k}$ is the highest labeled player that is on both paths. Further, define $m=\min _{h, k} m_{h k}$. Suppose $m \geq 1$, i.e., there exist two paths, say $p^{1}$ and $p^{2}$ with $H_{i}\left(p^{1}\right) \cap H_{i}\left(p^{2}\right) \neq \emptyset$, such that (i) $m$ is the highest labeled common node of $p^{1}$ and $p^{2}$ and (ii) $m_{h k} \geq m$ for any two paths $p^{h}$ and $p^{k}$ in $\mathcal{P}_{i}$. Since $i$ is a free player, and thus $\bar{P}_{D}(i)=\emptyset$, there is no player $j \neq i$ that is on all paths in $\mathcal{P}_{i}$. Therefore we must have a third path, say $p^{3} \in \mathcal{P}_{i}$, such that $m \notin H_{i}\left(p^{3}\right)$. Because of (ii) we have that $m_{13}>m$ and $m_{23}>m$, so for both paths $p^{1}$ and $p^{2}$ it holds that they have a node in common with $p^{3}$ with a higher label than $m$. For $j=1,2$, define $m_{j}=\min \left\{s>m \mid s \in H_{i}\left(p^{j}\right) \cap H_{i}\left(p^{3}\right)\right\}$, so $m_{j}$ is the lowest labeled common node on the paths $p^{j}$ and $p^{3}$ higher than $m$. Thus $m_{1}>m$ and $m_{2}>m$ and also $m_{1} \neq m_{2}$, otherwise $m_{1}=m_{2} \in H_{i}\left(p^{1}\right) \cap H_{i}\left(p^{2}\right)$, contradicting (i). Without loss of generality, suppose that $m_{1}>m_{2}$. Now, let $p^{4}$ be the path in $\mathcal{P}_{i}$ that is equal to $p^{3}$ from $T_{D}$ to node $m_{2}$ and it is equal to $p^{2}$ from $m_{2}$ to $i$. Then $m_{14}<m$, because $p^{4}$ coincides with $p^{2}$ from node $m_{2}$ to $i$ and the highest labeled common node of $p^{1}$ and $p^{2}$ is node $m<m_{2}$, and $p^{4}$ coincides with $p^{3}$ from $T_{D}$ to $m_{2}$ and the smallest labeled common node of $p^{1}$ and $p^{3}$ higher or equal to $m$ is node $m_{1}>m_{2}$. However, $m_{14}<m$ contradicts (ii). So it follows that $m=0$, which proves that there exists two paths in $\mathcal{P}_{i}$ that only have node $i$ in common.

Recall that in the first iteration player 1 is chosen to be the top and that the nucleolus payoffs of the players in $U^{1}$ are computed. In the second iteration the algorithm continues with the non-negative additive game with permission structure $\left(K, v_{K}, D^{K}\right)$, where $K=N \backslash U^{1},\left(K, v_{K}\right)$ the subgame of $(N, v)$ on $K$, and $D^{K}$ the permission structure as obtained in formula (3.2). The next lemma states that $j \neq 1$ is a free player in $\left(K, D^{K}\right)$ if and only if it is a free player in $(N, D)$.

Lemma 5.5 Let $(N, D)$ be an acyclic permission structure, $K=N \backslash U^{1}$ and $D^{K}$ the permission structure as defined in formula (3.2). Then $F_{D^{K}}=F_{D} \backslash\{1\}$.

Proof. First, observe that $U^{1}$ consists of player 1 and all its complete subordinates, so by Lemma 3.1, $U^{1}$ does not contain any of the players in $F_{D} \backslash\{1\}$, and thus $F_{D} \backslash\{1\} \subseteq K$. We first prove that $F_{D} \backslash\{1\} \subseteq F_{D_{K}}$. When $i \in T_{D} \backslash\{1\}$, then obviously $i \in T_{D^{K}}$. So, consider $i \in F_{D} \backslash T_{D}$. According to Lemma 5.4, there are two different paths in $(N, D)$ from $T_{D}$ to $i$. Let $p$ be such a path. We consider two cases.

1. The path $p$ is completely in $K$ and $P_{D}(j) \cap U^{1}=\emptyset$ for any $j \in H(p)$. Then any link $(h, k) \in D$ on the path is also a link in $D_{K}$ and $p^{\prime}=p$ is a path in $\left(K, D_{K}\right)$ from $T_{D} \backslash\{1\} \subseteq T_{D^{K}}$ to $i$.

2. There is some $h \in H(p)$ such that $P_{D}(h) \cap U^{1} \neq \emptyset$. With $A(p)$ the set of all players in $H(p)$ having a predecessor in $U^{1}$, let $k$ be the player in $A(p)$ such that $k \geq h$ for 
all $h \in A(p)$, i.e., $k$ is the player in $A(p)$ with the highest label. Then, by formula (3.2), any link $(i, k) \in D(K)$ is deleted to obtain $D^{K}$, so $k \in T_{D^{K}}$. Let $p^{\prime}$ be the path consisting of the part of path $p$ from $k$ to $i$. Then $p^{\prime}$ is a path from $T_{D^{K}}$ to $i$ in $(K, D(K))$. Observe that $p^{\prime}=(i)$ with $i \in T_{D^{K}}$ if $k=i$.

It follows that every path $p$ from $T_{D}$ to $i$ in $(N, D)$ gives a path $p^{\prime}$ from $T_{D^{K}}$ to $i$ in $(K, D(K))$. When for some $p$ the path $p^{\prime}$ reduces to the single player path $p^{\prime}=(i)$ (when $k=i$ in case 2$)$, then $i$ becomes a top player in $(K, D(K))$ and every path from $T_{D}$ to $i$ reduces to the single element $i$. Otherwise, $i$ has two different paths in $\left.\left(K, D_{K}\right)\right)$ when $i$ has two different paths in $(N, D)$, because $H\left(p^{\prime}\right) \subseteq H(p)$ for every path $p$ from $T_{D}$ to $i$. So $i \in F_{D^{K}}$ when $i \in F_{D} \backslash\{1\}$.

Second, we prove the reverse inclusion that $F_{D^{K}} \subseteq F_{D} \backslash\{1\}$. To do so we show that a node $i \neq 1$ which is not free in $(N, D)$ is also not free in $\left(K, D_{K}\right)$. Let $i \notin F_{D} \backslash\{1\}$, so $i$ has a complete superior in $(N, D)$. When there is a complete superior in $U^{1}$ then, by Lemma 5.3, also 1 is a complete superior of $i$. Then $i \in U^{1}$ and thus $i$ is not in $K$. It remains to consider the case that $\bar{P}_{D}(i) \subset K$. Let $k$ be a player in $\bar{P}_{D}(i)$. For $p$ a path from $T_{D}$ to $i$, let $p^{\prime}$ be the part of the path from $k$ to $i$. Then there is no player $h>k$ on the path $p^{\prime}$ that has a predecessor in $U^{1}$, otherwise there is in $(N, D)$ a path from 1 to the predecessor of $h$ in $U^{1}$, then to $h$ and then to $i$, contradicting that $k$ is a complete superior of $i$ in the graph $(N, D)$. So, when $(j, l) \in D$ is a link on $p^{\prime}$, then also $(j, l) \in D_{K}$ and thus $p^{\prime}$ is a path in $\left(K, D_{K}\right)$. Since this holds for every path $p$ from $T_{D}$ to $i$ in $(N, D)$, it follows that $k$ is also a complete superior of $i$ in $\left(K, D_{K}\right)$, and thus $i$ is not free in $\left(K, D_{K}\right)$.

The next lemma states that a player is chosen as top in one of the iterations of the algorithm if and only if it is a free player in $(N, D)$. Let $A_{D}$ denote the set of players that is chosen as top in one of the iterations of the algorithm of Section 3.

Lemma 5.6 Let $(N, v, D)$ be a non-negative additive game with acyclic permission structure. Then $A_{D}=F_{D}$.

Proof. The proof follows by repeated application of Lemma 5.5. In iteration 1, player 1 is chosen as top. So, player 1 belongs both to $A_{D}$ and $F_{D}$. In iteration 2 , player $t_{2}$ is chosen as top. This player is determined in Step 5 of the previous iteration as the top player with the lowest label in $\left(N_{2}, D_{2}\right)$ with $N_{2}=N_{1} \backslash U^{1}=N \backslash U^{1}$ and $D_{2}=D^{N_{2}}$. By Lemma 5.5 this top belongs to $F_{D_{2}}=F_{D} \backslash\{1\}$. From repeated application of the lemma it follows that the top $t_{k}$ in iteration $k$ is a top of $F_{D_{k}}=F_{D_{k-1}} \backslash\left\{t_{k-1}\right\}=F_{D} \backslash\left\{t_{1}, \ldots, t_{k-1}\right\}$, where $\left(N_{k}, D_{k}\right)$ is the graph at iteration $k$. It follows that succeedingly all players of $F_{D}$ are chosen as top in increasing order of their label.

Finally we show that the set of complete subordinates of the chosen top $t_{k}$ in the graph $\left(N_{k}, D_{k}\right)$ in iteration $k$ is equal to the set of complete subordinates of the free player 
$t_{k}$ in $(N, D)$. Moreover we have that $\left(U^{t_{k}}, D_{k}\left(U^{t_{k}}\right)\right)=\left(U^{t_{k}}, D\left(U^{t_{k}}\right)\right)$, i.e., the subgraph on $U^{t_{k}}$ of $\left(N_{k}, D_{k}\right)$ is equal to the subgraph of $U^{t_{k}}$ of $(N, D)$.

Lemma 5.7 Let $(N, v, D)$ be a non-negative additive game with acyclic permission structure and let $t_{k}$ be the chosen top in iteration $k$ of the algorithm. Then $\bar{S}_{D_{k}}\left(t_{k}\right)=\bar{S}_{D}\left(t_{k}\right)$. Moreover, $\left(U^{t_{k}}, D_{k}\left(U^{t_{k}}\right)\right)=\left(U^{t_{k}}, D\left(U^{t_{k}}\right)\right)$.

Proof. From the proof of Lemma 5.5 it follows that when $k \in N_{2}=N_{1} \backslash U^{1}$ is a complete superior of $i$ in $\left(N_{1}, D_{1}\right)=(N, D)$, then $k$ is also a complete superior of $i$ in $\left(N_{2}, D_{2}\right)$. With Lemma 3.1 it follows reversely that $i \in \bar{S}_{D_{2}}(k)$ when $i \in \bar{S}_{D_{1}}(k)$. On the other hand, when $i$ is not a complete subordinate of $k$ in $\left(N_{1}, D_{1}\right)$, then it also not in $\left(N_{2}, D_{2}\right)$ because $D_{2} \subset D_{1}$ and either $P_{D_{2}}(i)=\emptyset$ or there is a path from $T_{D_{2}}$ to $i$ in $\left(N_{2}, D_{2}\right)$ without $k$. So, for any $k \in N_{2}$ we have that $\bar{S}_{D_{2}}(k)=\bar{S}_{D_{1}}(k)$. The first statement of the lemma follows by repeating these arguments for all the remaining top players at any iteration of the algorithm. To show the second statement, let $k \in N_{2}$ be a complete superior of a player $i \in N_{2}$. From the last part of the proof of Lemma 5.5 it follows that any link on a path from $k$ to $i$ in $\left(N_{1}, D_{1}\right)$ is also a link in $\left(N_{2}, D_{2}\right)$. So, $\left(U^{t_{2}}, D_{2}\left(U^{t_{2}}\right)\right)=\left(U^{t_{2}}, D_{1}\left(U^{t_{2}}\right)\right)=\left(U^{t_{2}}, D\left(U^{t_{2}}\right)\right)$. The result follows from repeating this at any next iteration of the algorithm.

We now come to the main result of this section. In Section 4 we have seen that the algorithm of Section 3 at any iteration $k$ computes the nucleolus payoffs in the restricted game $(N, r)$ of the players in $U^{t_{k}}$, where $U^{t_{k}}$ is the set of players consisting of the chosen top $t_{k}$ in iteration $k$ and all its complete subordinates in $\left(N_{k}, D_{k}\right)$. Moreover, the total payoff of the players in $U^{t_{k}}$ is equal to the sum of their values $a_{i}, i \in U^{t_{k}}$. Therefore the next proposition follows from the lemmas above without further proof. It states that for any free player the nucleolus distributes the total contributions of this player and its complete subordinates among themselves.

Proposition 5.8 Let $(N, v, D)$ be a non-negative additive game with acyclic permission structure. Then $\sum_{i \in \bar{S}_{D}(k)} N u c_{i}(N, r)=\sum_{i \in \bar{S}_{D}(k)} a_{i}$ for every $k \in F_{D}$.

Finally, when the structure of the graph $(N, D)$ is known ex ante, in particular the set $F_{D}$ is known ex ante and also for each $k \in F_{D}$ its set of complete subordinates, it follows without further proof from the lemmas above that the algorithm of Section 3 reduces to $\left|F_{D}\right|$ applications of the algorithm of van den Brink et al. (2008). To state this result, for $k \in F_{D}$, let $\bar{r}_{k}$ be the restricted game of $\left(\bar{S}_{D}(k), v_{k}, D\left(\bar{S}_{D}(k)\right)\right.$, where $v_{k}$ is the non-negative additive game on $\bar{S}_{D}(k)$ given by $v_{k}(S)=\sum_{i \in S} a_{i}, S \subseteq \bar{S}_{D}(k)$. Then we have the following proposition. 
Proposition 5.9 Let $(N, v, D)$ be a non-negative additive game with acyclic permission structure. Then $N u c_{i}(N, r)=N u c_{i}\left(\bar{S}_{D}(k), r_{k}\right), i \in \bar{S}_{D}(k), k \in F_{D}$.

From this last proposition we can conclude that for any $k \in F_{D}$, the nucleolus values in the game $(N, r)$ of the players in $\bar{S}_{D}(k)$ can be computed by applying the algorithm of van den Brink et al. (2008) to $\left(\bar{S}_{D}(k), v_{k}, D\left(\bar{S}_{D}(k)\right)\right.$.

\section{Complexity of the algorithm}

When the structure of the game is known ex ante we can apply Proposition 5.9 and apply the algorithm of van den Brink et al. (2008) to any $\left(\bar{S}_{D}(k), v_{k}, D\left(\bar{S}_{D}(k)\right), k \in F_{D}\right.$. Since the complexity of this algorithm is of $\mathcal{O}\left(n^{4}\right)$, the complexity reduces to $\mathcal{O}\left(\left|F_{D}\right| a^{4}\right)$, where $a=\max _{k \in F_{D}}\left|\bar{S}_{D}(k)\right|$, because we have $\left|F_{D}\right|$ problems and the $k$ th problem has complexity $\mathcal{O}\left(\left|\bar{S}_{D}(k)\right|^{4}\right)$. In particular, we have that the complexity is of $\mathcal{O}\left(n^{4}\right)$ when $\left|F_{D}\right|=1$ (and thus $\left|\bar{S}_{D}(1)\right|=n$ ) and of $\mathcal{O}(n)$ when $\left|F_{D}\right|=n$ (and thus $\left|\bar{S}_{D}(k)\right|=1$ for all $k$ ). Clearly, in the latter (extreme) case we have that $(N, D)$ is the empty graph and $(N, v, D)$ reduces to the additive game, so that every player $i$ gets its own value $a_{i}$.

Typically in practice the structure is not known in advance. Also, although we assumed in the previous sections that the players are enumerated by the numbers $1,2, \ldots, n$ in such a way that for any $i, j \in\{1, \ldots, n\}$ it holds that $i<j$ if $(i, j) \in D$, in practice such an enumeration will not be known in advance. So, to perform the algorithm at each iteration $k$ first a top node $t_{k}$ in $\left(N_{k}, D_{k}\right)$ has to be found and its corresponding set $U^{t_{k}}$ of complete subordinates in $\left(N_{k}, D_{k}\right)$. For the complexity of this search, let us consider the first iteration. It is evident that we can find the collection $T_{D}$ of top nodes in at most $n^{2}$ actions, because we just have to consider each pair of players once. Then we enumerate the top nodes from 1 to $k_{1}$, where $k_{1}=\left|T_{D}\right|$ is the number of top nodes in $(N, D)$. To find the set $U^{1}$ of all complete subordinates of top node 1 we can proceed as follows. First, assign label 1 to every successor of node 1 . Next, assign label 1 to every successor of every node with label 1 and continu to do this. So, every player in the set $\widehat{S}_{D}(1)$ of subordinates of top player 1 gets a label 1 . Clearly, this requires at most $\mathcal{O}\left(n^{2}\right)$ actions. Next, repeat this procedure for every other top node, so for every player in the set $\widehat{S}_{D}(j)$ of top node $j, j=1, \ldots, k_{1}$. So, a node can receive multiple labels. The set $U^{1}$ consists of all nodes that only receive label 1 and can be found in at most $k_{1} \mathcal{O}\left(n^{2}\right)<\mathcal{O}\left(n^{3}\right)$ actions. In fact this procedure gives in at most $\mathcal{O}\left(n^{3}\right)$ actions all top nodes $j \in T_{D}$ and their sets $U^{j}$ of complete subordinates.

We can now use the result of Proposition 5.9 to modify the algorithm, namely instead of adapting the graph after the first iteration according to Step 5, we first apply $k_{1}$ times the algorithm of van den Brink et al. (2008), namely to subgame with permission 
structure $\left(U^{k}, v_{k}, D\left(U^{k}\right)\right)$ for every top player $k \in T_{D}$. The complexity of each application is given by $\mathcal{O}\left(\left|U^{k}\right|^{4}\right)$.

After having eliminated all top players and their complete subordinates we now adapt the graph on the remaining set of players $N \backslash\left(\cup_{k \in T_{D}} U^{k}\right)$ analogously as described in Step 5 of the algorithm. Let $M$ be this set, then for each player $j \in M$ we consider whether or not $j$ has a predecessor in $(N, D)$ belonging to $\cup_{k \in T_{D}} U^{k}$. If so, all edges $(h, j)$ in the subgraph $(M, D(M))$ are deleted and $j$ becomes a top node in the remaining graph. Observe that such a top node in the new graph is a free player in $(N, D)$. This requires at most $\mathcal{O}\left(n^{2}\right)$ actions and yields also the set of top nodes in the remaining graph. Next, repeating the procedure as described above for the set of new top nodes, in $\mathcal{O}\left(\mathrm{m}^{3}\right)$ actions, where $m=|M|$, the sets $U^{j}$ of complete subordinates of the new top nodes can be found. Then we apply again the algorithm of van den Brink et al. (2008) to each new top node $j$ and its set of complete subordinates $U^{j}$. After that we apply the procedure of finding the new graph and new set of top players for the remaining nodes and so on.

Summarizing, starting the algorithm the number of actions to find the set of top nodes and their sets of complete subordinates is of $\mathcal{O}\left(n^{3}\right)$. This has to be repeated at most $n-1$ times to find the set of all free players (each free player is a top node at some stage) and their sets of complete subordinates. So, the number of actions to find all free players and their sets of complete subordinates is (at most) of $\mathcal{O}\left(n^{4}\right)$. For each free player $k \in F_{D}$ the complexity of the algorithm of van den Brink et al. is of $\mathcal{O}\left(\left|U^{k}\right|^{4}\right)$. Since $\sum_{k=1}^{s}\left|U^{k}\right|=n$, where $s=\left|F_{D}\right|$, the total complexity of applying the algorithm $s$ times, namely for each free player, is of $\mathcal{O}\left(n^{4}\right)$. So, both the complexity of finding all free players and their sets of complete subordinates and the total complexity to find the nucleolus payoffs for every free player with its set of complete subordinates, is given by $\mathcal{O}\left(n^{4}\right)$. Hence the total complexity of the algorithm of Section 3 to find all nucleolus payoffs is of $\mathcal{O}\left(n^{4}\right)$, showing that the algorithm finds the nucleolus in polynomial time.

\section{An example}

In this section we illustrate the computation of the nucleolus for non-negative additive disjunctive games with a permission structure by giving an example concerning a market situation where sellers can sell objects to buyers through a (directed) network of intermediaries. First, we give a simple example without intermediaries ${ }^{7}$.

Example 7.1 Consider a situation where there is one seller (player 1) and one buyer (player 2) who can realize a non-negative surplus $a>0$ from trade. The corresponding

\footnotetext{
${ }^{7}$ This is a special case of the assignment game, introduced by Shapley and Shubik (1972).
} 
assignment game on $N=\{1,2\}$ is given by $v(\{1\})=v(\{2\})=0$ and $v(\{1,2\})=a$. Note that the restricted game $(N, r)$ on the permission structure $D=\{(1,2)\}$ (or $D=\{(2,1)\}$ ) is the same as $(N, v)$. Clearly, the nucleolus of this game yields an equal division of the surplus, i.e., $N u c(N, v)=N u c(N, r)=\left(\frac{a}{2}, \frac{a}{2}\right)$. But as soon as there are two or more sellers (say players $1, \ldots, n-1\}$, such that the buyer (player $n$ ) can realize the surplus $a$ with any one of the sellers and cannot generate more surplus by trading with more sellers (for example, the sellers all own one item of a good for which they have reservation value zero, and the buyer wants only one item of the good and is prepared to pay at most $a$ for it) then the characteristic function of the assignment game on $N=\{1, \ldots n\}$ is $v(S)=a$ if $n \in S$ and $|S| \geq 2$, and $v(S)=0$ otherwise. Again the restricted game on the permission structure $D=\{(i, n) \mid i \in\{1, \ldots n-1\}$ (i.e., the permission structure where all sellers are predecessor of the buyer) is the same as $(N, v)$. Now, it is clear that $N u c_{n}(N, v)=N u c_{n}(N, r)=a$ and $N u c_{i}(N, v)=N u c_{i}(N, r)=0$ for all $i \in\{1, \ldots n-1\}$ since this is the unique Core payoff vector. This also follows immediately from Proposition 5.8 by observing that $r$ is the restricted game of the non-negative additive game with $a_{i}=0$ for $i=1, \ldots, n_{1}$ and $a_{n}=a$ and that player $n$ is a free player in $(N, D)$ and thus receives its own value. So, similar as in a linear Bertrand price competition game, as soon as there is more than one seller, the surplus fully goes to the buyer. (A similar story holds if there is only one seller but more buyers.)

Next, we consider an example of a market situation as described above, but buyers and sellers may not be able to trade directly with each other, but need intermediaries to connect them.

Example 7.2 Consider a market situation with two sellers (players 1 and 2) and four buyers (players 7, 8, 9 and 10) who cannot trade directly with each other but need intermediaries. Consider the permission structure $D$ on $N=\{1, \ldots, 10\}$ given by $D=$ $\{(1,3),(1,4),(2,4),(2,5),(2,6),(3,7),(4,7),(4,8),(5,9),(6,9),(6,10)\}$, see Figure 1. For every buyer-seller pair that wants to make a deal, it is sufficient to use only one of the intermediaries they are both connected with. For example, for seller 1 and buyer 7 it is necessary and sufficient to use either intermediary 3 or intermediary 4, while seller 1 and buyer 8 need intermediary 4 to trade. Suppose that each seller owns at least four items of the good and each buyer wants one item. Buyer $i \in\{7,8,9,10\}$ is prepared to pay $a_{i}>0$ for the item. The reservation value of the sellers and all intermediaries is zero. This can be modelled as the game with permission structure $(N, v, D)$ with $N=\{1, \ldots, 10\}, D$ as given above, and $v$ the non-negative additive game with $a_{i}=0$ for $i=1, \ldots, 6$ and $a_{i}>0$ for $i=7, \ldots, 10$, so that $v(S)=\sum_{i \in S} a_{i}=\sum_{i \in S \cap\{7,8,9,10\}} a_{i}$ for all $S \subseteq N$. 


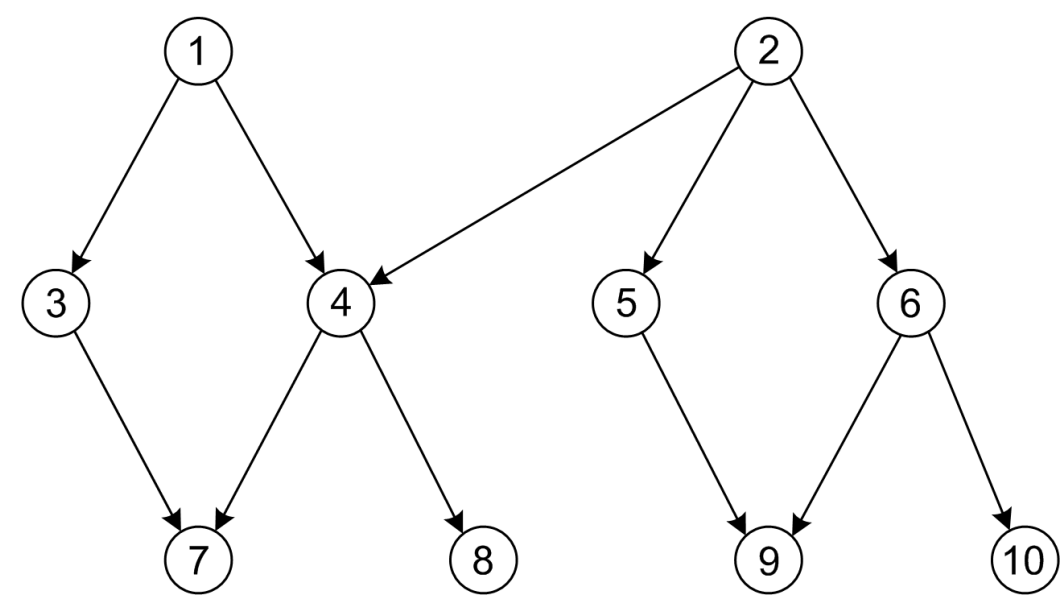

Figure 1.

The nucleolus of the restricted game $(N, r)$ can be computed using the algorithm of Section 3 and the properties of Section 5 . Notice that the players 1 and 2 are top players, and that the set of free players is given by $F_{D}=\{1,2,4,7\}$. Using Proposition 5.9 it follows that we can find the nucleolus of $(N, r)$ by considering the four subgames with permission structure given in Figure 2, namely one subgame for each of the four free players.
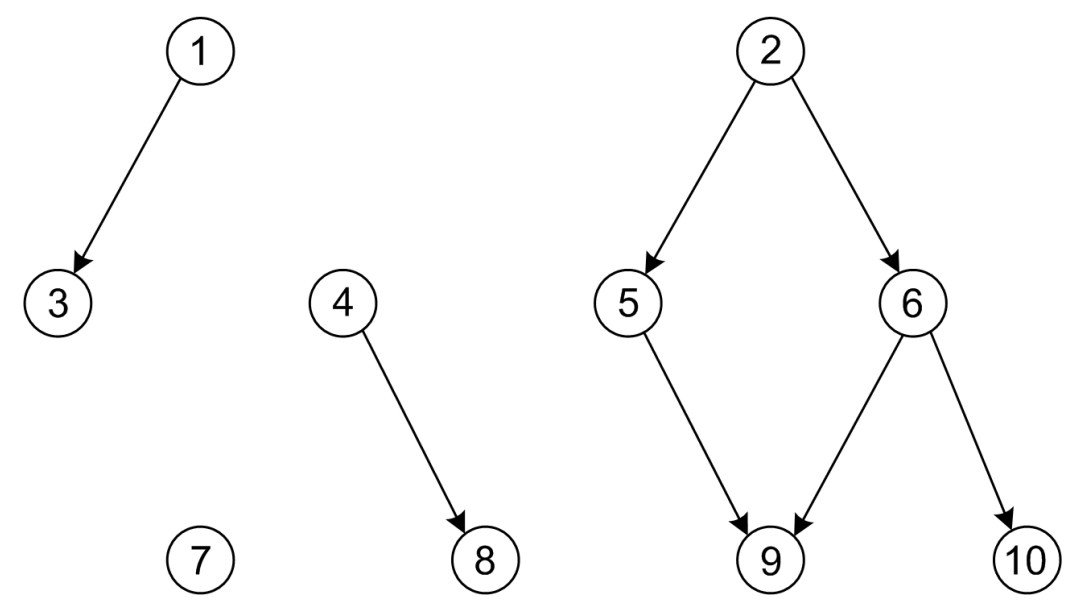

Figure 2 
The nucleoli for the four subgames are as follows.

1. Consider the game with permission structure $\left(N_{1}, v_{1}, D_{1}\right)$ given by $N_{1}=\{1,3\}, v_{1}=$ $\left.v\right|_{N_{1}}$ and $D_{1}=\left.D\right|_{N_{1}}=\{(1,3)\}$. Since $v_{1}$ is the null game assigning worth zero to all coalitions in $N_{1}$, it follows that $N u c_{1}\left(N_{1}, r_{1}\right)=N u c_{3}\left(N_{1}, r_{1}\right)=0$.

2. For the game with permission structure $\left(N_{2}, v_{2}, D_{2}\right)$ given by $N_{2}=\{2,5,6,9,10\}, v_{2}=$ $\left.v\right|_{N_{2}}$, and $D_{2}=\left.D\right|_{N_{2}}=\{(2,5),(2,6),(5,9),(6,9),(6,10)\}$, we have that $v_{2}(S)=$ $\sum_{i \in S \cap\{9,10\}} a_{i}$ for all $S \subseteq N$. Then the restricted game is given by

$$
r_{2}(S)=\left\{\begin{array}{cl}
a_{9} & \text { if } S \in\{\{2,5,9\},\{2,6,9\},\{2,5,6,9\}\} \\
a_{10} & \text { if } S \in\{\{2,6,10\},\{2,5,6,10\}\} \\
a_{9}+a_{10} & \text { if } S \in\{\{2,6,9,10\},\{2,5,6,9,10\}\} \\
0 & \text { otherwise. }
\end{array}\right.
$$

Applying the algorithm of van den Brink et al. (2008) (see the Appendix) we find for any pair of values $a_{9}, a_{10} \geq 0$ that $N u c_{2}\left(N_{2}, r_{2}\right)=\frac{a_{9}}{2}+\frac{a_{10}}{3}, N u c_{5}\left(N_{2}, r_{2}\right)=$ $0, N u c_{6}\left(N_{2}, r_{2}\right)=\frac{a_{10}}{3}, N u c_{9}\left(N_{2}, r_{2}\right)=\frac{a_{9}}{2}$ and $N u c_{10}\left(N_{2}, r_{2}\right)=\frac{a_{10}}{3}$.

3. For the game with permission structure $\left(N_{3}, v_{3}, D_{3}\right)$ given by $N_{3}=\{4,8\}, v_{3}=\left.v\right|_{N_{3}}$ and $D_{3}=\left.D\right|_{N_{3}}=\{(4,8)\}$, we have that $v_{3}(S)=\sum_{i \in S \cap\{8\}} a_{i}$ for all $S \subseteq N$. So, the restricted game is given by

$$
r_{3}(S)=\left\{\begin{array}{cl}
a_{8} & \text { if } S=\{4,8\} \\
0 & \text { otherwise. }
\end{array}\right.
$$

We find that $N u c_{4}\left(N_{3}, r_{3}\right)=N u c_{8}\left(N_{3}, r_{3}\right)=\frac{a_{8}}{2}$.

4. The game with permission structure $\left(N_{4}, v_{4}, D_{4}\right)$ is given by $N_{4}=\{7\}, v_{4}=\left.v\right|_{N_{4}}$ and $D_{4}=\emptyset$. Clearly $v_{4}(S)=r_{4}(S)=a_{7}$ for $S=\{7\}$, and thus $N u c_{7}\left(N_{4}, r_{4}\right)=a_{7}$.

By Proposition 5.9 we then have $N u c(N, r)=\left(0, \frac{a_{9}}{2}+\frac{a_{10}}{3}, 0, \frac{a_{8}}{2}, 0, \frac{a_{10}}{3}, a_{7}, \frac{a_{8}}{2}, \frac{a_{9}}{2}, \frac{a_{10}}{3}\right)$.

In the example above, there was only one level of intermediaries, but the algorithm also works if there are more levels of intermediaries between buyers and sellers.

\section{Appendix: A polynomial time algorithm to compute the nucleolus of a non-negative additive game with an acyclic, quasi-strongly connected permission structure}

In this appendix we recall the polynomial time algorithm of van den Brink et al. (2008). Although they use this algorithm to compute the nucleolus for a more general class of games 
(those that satisfy the so-called weak digraph monotonicity and weak digraph convexity conditions) we only apply it to non-negative additive games with an acyclic, quasi-strongly connected permission structure in step 3 of the algorithm to compute the nucleolus for nonnegative additive games with a (more general) acyclic permission structure. This algorithm computes the nucleolus in $\mathcal{O}\left(n^{4}\right)$ time. It is an adaptation of the algorithm of Arin and Feltkamp (1997) which computes the nucleolus of veto-rich games in exponential time. In the following, $\Omega^{D}=\Phi^{D} \backslash\{N\}$ denotes the collection of all feasible coalitions not equal to $N$.

Consider a given non-negative additive game with an acyclic, quasi-strongly connected permission structure $(N, v, D)$.

\section{Algorithm}

Step 1 Set $k=0, U_{0}=N, v_{0}=v, D_{0}=D$ and $r_{0}=r$. Goto Step 2 .

Step 2 Find $U_{k+1} \subset U_{k}$ satisfying

$$
\tau\left(U_{k+1}, r_{k}\right)=\tau^{*}\left(r_{k}\right) \text { and }\left|U_{k+1}\right|=\max _{\left\{U \in \Omega^{D_{k}} \mid \tau\left(U, r_{k}\right)=\tau^{*}\left(r_{k}\right)\right\}}|U|
$$

where $\tau^{*}\left(r_{k}\right)=\min _{U \in \Omega^{D_{k}}} \tau\left(U, r_{k}\right)$ with $\tau\left(U, r_{k}\right)=\frac{r_{k}\left(U_{k}\right)-r_{k}(U)}{\left|U_{k} \backslash U\right|+1}$. Assign $y_{j}=\tau^{*}\left(r_{k}\right)$ to every player $j \in U_{k} \backslash U_{k+1}$. Goto Step 3 .

Step 3 If $U_{k+1}=\{1\}$ then Goto Step 4. If $U_{k+1} \neq\{1\}$, let $i_{k+1}$ be the unique top-player of the subgraph $\left(U_{k} \backslash U_{k+1}, D_{k}\left(U_{k} \backslash U_{k+1}\right)\right.$ of the digraph $\left(U_{k}, D_{k}\right)$ restricted to $U_{k} \backslash U_{k+1}$. Define game $\left(U_{k+1}, v_{k+1}\right)$ by setting for every $U \subseteq U_{k+1}$,

$v_{k+1}(U)= \begin{cases}v_{k}(U) & \text { if } P_{D_{k}}\left(i_{k+1}\right) \cap U=\emptyset \\ v_{k}\left(U \cup\left(U_{k} \backslash U_{k+1}\right)\right)-\tau\left(U_{k+1}, r_{k}\right)\left|U_{k} \backslash U_{k+1}\right| & \text { else, }\end{cases}$

digraph $\left(U_{k+1}, D_{k+1}\right)$ given by

$$
(i, j) \in D_{k+1} \text { if }\left\{\begin{array}{l}
(i, j) \in D_{k} \text { or } \\
U_{k+1}
\end{array}\right.
$$

and let $r_{k+1}$ be the restricted game of $\left(U_{k+1}, v_{k+1}, D_{k+1}\right)$. Set $k=k+1$. Goto Step 2 .

Step 4 Assign $y_{1}=v(N)-\sum_{j \in N \backslash\{1\}} x_{j}$. Stop. 


\section{References}

Arin, J., and Feltkamp, V. (1997), The nucleolus and kernel of veto-rich transferable utility games, International Journal of Game Theory 26, 61-73.

Bilbao, J.M., Jiménez-Losada, A., Lebrón, E., and Chacón, C. (2005), Values for interior operator games, Annals of Operations Research, 137, 141-160.

Bilbao, J.M., Chacón, C., Jiménez-Losada, A., and Lebrón, E. (2008), Convexity properties for interior operator games, Annals of Operations Research, 158, 117-131.

Bondareva, O. (1962), The theory of the Core in an $n$-person game, Vestnik Leningrad. Univ. 13, 141-142 (in Russian).

Brânzei, R., Fragnelli, V., and Tijs, S. (2002), Tree connected line graph peer group situations and line graph peer group games, Mathematical Methods of Operations Research 55, 93-106.

Brânzei, R., Solymosi, T., and Tijs, S. (2005), Strongly essential coalitions and the nucleolus of peer group games, International Journal of Game Theory 33, 447-460.

Brink, R. van den (1997), An axiomatization of the disjunctive permission value for games with a permission structure, International Journal of Game Theory 26, 27-43.

Brink, R. van den, and Gilles, R. P. (1996), Axiomatizations of the conjunctive permission value for games with permission structures, Games and Economic Behavior 12, 113126.

Brink, R. van den, I. Katsev and G. van der Laan (2008), Computation of the nucleolus for a class of disjunctive games with a permission structure, Discussion Paper TI 08-060/1, Tinbergen Institute, Amsterdam/Rotterdam, The Netherlands.

Brune, S. (1983), On the regions of linearity for the nucleolus and their computation, International Journal of Game Theory 12, 47-80.

Gilles, R. P., and Owen, G. (1994), Cooperative games and disjunctive permission structures, Department of Economics, Virginia Polytechnic Institute and State University, Blacksburg, Virginia.

Gilles, R. P., Owen, G., and Brink, R. van den (1992), Games with permission structures: the conjunctive approach, International Journal of Game Theory 20, 277-293. 
Gillies, D.B. (1953), Some Theorems on n-Person Games, Princeton University Press, Princeton, NJ.

Huberman, G. (1980), The nucleolus and essential coalitions, in: A. Bensoussan and J. Lions (eds.) Analysis and Optimization of Systems, Lecture Notes in Control and Information Sciences 28, Springer, Berlin, pp. 416-422.

Kohlberg, E., (1979), On the nucleolus of a characteristic function game, SIAM Journal of Applied Mathematics 20, 62-66.

Muto, S., Potters, J., and Tijs, S. (1989), Information market games, International Journal of Game Theory 18, 209-226.

Myerson, R. B. (1977), Graphs and cooperation in games, Mathematics of Operations Research 2, 225-229.

Ni, D., and Y. Wang (2007), Sharing a polluted river, Games and Economic Behavior, 60, 176-186.

Reynierse, J., and Potters, J. (1998), The $\mathcal{B}$-nucleolus of TU-games, Games and Economic Behavior 24, 77-96.

Schmeidler, D. (1969), The nucleolus of a characteristic function game, SIAM Journal on Applied Mathematics 17, 1163-1170.

Shapley, L. S. (1953), A value for N-person games, in: Contributions to the Theory of Games, Vol II (eds. H. W. Kuhn, and A. W. Tucker), Princeton University Press, Princeton, 307-317.

Shapley, L.S. (1967), On balanced sets and cores, Naval Research Logistics Quarterly 14, 453-460.

Shapley, L., and M. Shubik (1972), The assignment game I: the core, International Journal of Game Theory, 1, 111-130. 